\title{
DETECTION OF COOL DUST AROUND THE G2 V STAR HD 107146
}

\author{
Jonathan P. Williams, ${ }^{1}$ Joan Najita, ${ }^{2}$ Michael C. Liu, ${ }^{1,3}$ Sandrine Bottinelli, ${ }^{1}$ John M. Carpenter, \\ Lynne A. Hillenbrand, ${ }^{4}$ Michael R. Meyer, ${ }^{5}$ and David R. Soderblom ${ }^{6}$ \\ Received 2003 May 13; accepted 2003 December 1
}

\begin{abstract}
We report the detection of dust emission at submillimeter wavelengths from HD 107146, a G2 V star with an age estimated to lie between 80 and $200 \mathrm{Myr}$. The emission is resolved at $450 \mu \mathrm{m}$ with a size $300 \mathrm{AU} \times 210 \mathrm{AU}$. A fit to the spectral energy distribution gives a dust temperature of $51 \mathrm{~K}$ and a dust mass of $0.10 M_{\oplus}$. No excess emission above the photosphere was detected at $18 \mu \mathrm{m}$, showing that there is very little warm dust and implying the presence of a large inner hole, at least $31 \mathrm{AU}\left(\sim 1^{\prime \prime}\right)$ in radius, around the star. The properties of this star-disk system are compared with similar observations of other systems. We also discuss prospects for future observations that may be able to determine whether the inner hole is maintained by the dynamical effect of an unseen orbiting companion.
\end{abstract}

Subject headings: circumstellar matter — planetary systems — stars: individual (HD 107146)

On-line material: color figure

\section{INTRODUCTION}

Protostellar disks dissipate over a period of several Myr as their constituent dust and gas either accrete onto the star, are dispersed by processes such as stellar winds and photoevaporation, or aggregate into planetesimals (Hollenbach, Yorke, \& Johnstone 2000). Possible observational examples of disk systems in the process of dissipating are the "transitional disks," a thus far small class of objects that includes sources such as V819 Tau (Skrutskie et al. 1990), HR 4796A (Jura et al. 1993; Jayawardhana et al. 1998; Koerner et al. 1998; Schneider et al. 1999; Telesco et al. 2000), and HD 141569A (Weinberger et al. 1999; Augereau et al. 1999; Fisher et al. 2000).

Dramatic evidence for disk dispersal through planetesimal formation is the detection of numerous planets, and some planetary systems, around nearby stars (Marcy, Cochran, \& Mayor 2000). Planetesimals are believed to grow into planets through collisional agglomeration. However, collisions between planetesimals are also expected to create a cascade of smaller particles, and in this way a relatively old circumstellar disk may regenerate its own dust.

Such second-generation dust was first detected around main-sequence stars by IRAS (Aumann et al. 1984). The IRAS results indicated that $\sim 15 \%$ of main-sequence stars possess dusty disks (Lagrange, Backman, \& Artymowicz 2000), the brightest of which have been detected at submillimeter wavelengths (Zuckerman \& Becklin 1993; Greaves et al. 1998; Sylvester, Dunkin, \& Barlow 2001; see also Wyatt,

\footnotetext{
${ }^{1}$ Institute for Astronomy, University of Hawaii, 2680 Woodlawn Drive, Honolulu, HI 96822; jpw@ifa.hawaii.edu, mliu@ifa.hawaii.edu, sandrine@ifa .hawaii.edu.

2 National Optical Astronomy Observatory, 950 North Cherry Avenue, Tucson, AZ 85719; najita@noao.edu.

${ }^{3}$ Hubble Fellow.

4 Department of Astronomy and Astrophysics, MS 105-24, California Institute of Technology, 1201 East California Boulevard, Pasadena, CA 91125; jmc@astro.caltech.edu, lah@astro.caltech.edu.

5 Steward Observatory, University of Arizona, 933 North Cherry Avenue, Tucson, AZ 85721; mmeyer@as.arizona.edu.

6 Space Telescope Science Institute, 3700 San Martin Drive, Baltimore, MD 21218; drs@stsci.edu.
}

Dent, \& Greaves 2003). Submillimeter observations are useful because they place strong constraints on the dust mass of the disk, because of the low optical depth of dust grains in the submillimeter compared with their opacities at shorter wavelengths. Because of their small angular size and weak emission, only four debris disk systems have been spatially resolved at these wavelengths to date (Holland et al. 1998; Greaves et al. 1998). The maps of these systems show interesting asymmetries that have been interpreted as the dynamical signature of a planetary companion (Holland et al. 2003; Wilner et al. 2002; Greaves et al. 1998).

Although the majority of the well-studied debris (and transitional) disk systems surround early-type stars (Vega, Fomalhaut, $\beta$ Pic, HR 4796A, and HD 141569A are all A stars), the expectation is that the process of disk dissipation and the regeneration of dust through planetesimal collisions is also a part of the evolutionary history of lower mass stars and, indeed, our own solar system. Thus, it is of considerable interest to identify debris disks associated with young solarmass stars, since the detailed study of these objects can provide direct insight into the evolutionary history of our solar system. The $\epsilon$ Eri system $(d=3.2 \mathrm{pc})$ is one exciting example (Greaves et al. 1998; Hatzes et al. 2000). In this paper, we present submillimeter observations of a dusty disk around a close solar analog, the nearby young G2 V star HD 107146.

HD 107146 was first identified as an "excess dwarf" on the basis of IRAS colors by Silverstone (2000). We observed it as part of a program to provide ground-based support for the Formation and Evolution of Planetary Systems Spitzer Space Telescope Legacy program. ${ }^{7}$ HD 107146 was selected for inclusion in the Legacy program based on its distance (28.5 pc; Perryman et al. 1997) and its high $\mathrm{Ca}$ II $\mathrm{H}$ and $\mathrm{K}$ index, $\log R_{\mathrm{HK}}^{\prime}=-4.28$, which is indicative of youth. An age range can be estimated from its lithium equivalent width, $125 \mathrm{~m} \AA$ (Wichmann, Schmitt, \& Hubrig 2003), which places it at the lower envelope of the $125 \mathrm{Myr}$ Pleiades distribution

\footnotetext{
${ }^{7}$ Information concerning this program can be found at http://feps.as arizona.edu.
} 
(Soderblom et al. 1993) but above the distribution for the 250 Myr old M34 (Jones et al. 1997) at the effective temperature of a G2 V star. Wichmann et al. also show that its space motions are similar to those of the Pleiades moving group. Its X-ray luminosity, $L_{\mathrm{X}}=2 \times 10^{29} \mathrm{ergs} \mathrm{s}^{-1}$ (Voges et al. 1999), is again similar to those of solar-type Pleiades stars (Micela et al. 1999) but weaker than that of the average solar-type stars in the $80 \mathrm{Myr}$ old $\alpha$ Per cluster (Prosser et al. 1996). Together, these indicators suggest an age for HD 107146 between 80 and 200 Myr. Its location in the H-R diagram is also consistent with this age range but allows for the possibility that it may be as young as $30 \mathrm{Myr}$ if the star has just reached the main sequence.

Our observations, made at submillimeter and mid-IR wavelengths, are detailed in $\S 2$. The results from the imaging and fits to the spectral energy distribution (SED) are presented in $\S 3$. The SED shows clear evidence for a large inner hole, although the hole is too small to be resolved in the submillimeter maps presented here. In $\S 4$, we compare the properties of the HD 107146 star-disk system to those of other debris and transitional disk systems. We also discuss prospects for future observations that may be able to determine whether the inner hole is maintained by the dynamical effect of an unseen orbiting companion.

\section{OBSERVATIONS}

HD 107146 was mapped using the Submillimeter CommonUser Bolometric Array (SCUBA) at the James Clerk Maxwell Telescope (JCMT) on Mauna Kea, Hawaii, in dry, stable conditions during 2003 February 17-19. Precipitable water vapor levels were less than $0.5-1 \mathrm{~mm}$ during the observations and zenith optical depths ranged from 0.1 to 0.18 at $850 \mu \mathrm{m}$ and from 0.4 to 0.85 at $450 \mu \mathrm{m}$. Observations were made simultaneously at 450 and $850 \mu \mathrm{m}$ using a 64 point "jiggle" pattern to produce Nyquist-sampled images at each wavelength. Two maps were rejected in the reduction process because of an error in the telescope tracking software at high elevation (I. Coulson 2003, private communication). The final maps presented here are the median of 23 maps made at a range of image rotation angles on the array rebinned to a $1^{\prime \prime}$ rectangular grid in equatorial coordinates. Pointing was checked between individual maps using Virgo A and 3C 273 and was accurate to an rms error of 2".5. Calibration was carried out by observing the CRL 618 planetary nebula and bootstrapped to observations of Mars later in the night. Based on the gain variations from night to night, the calibration accuracy is estimated to be $20 \%$ at $850 \mu \mathrm{m}$ and $30 \%$ at $450 \mu \mathrm{m}$. The total on-source integration time was $4.0 \mathrm{hr}$, and the noise in the final maps was 4 and $13 \mathrm{mJy}$ beam $^{-1}$ at 850 and $450 \mu \mathrm{m}$, respectively. The resulting peak signal-to-noise ratio is $\sim 7$ in both maps, but the calibration uncertainty dominates the error in the absolute flux measurements.

We obtained mid-IR photometry of HD 107146 from the Keck II Telescope on Mauna Kea, Hawaii, using the facility instrument LWS. We observed the star on 2003 February 19 and 20 UT, using filters centered at $11.7 \mu \mathrm{m}(10.5-12.9 \mu \mathrm{m})$ and $17.8 \mu \mathrm{m}(17.3-18.2 \mu \mathrm{m})$. Contemporaneously and at similar airmasses, we observed the bright standard stars $\alpha \mathrm{CrB}$ and $\mu$ UMa from Tokunaga (1988) for photometric calibration. Both nights were characterized by dry conditions, with an estimated $0.5-1.5 \mathrm{~mm}$ of precipitable water, as determined from sky dip measurements from the JCMT. Seeing conditions were poor and variable, leading to mid-IR images with 0 ".4
TABLE 1

Flux Measurements

\begin{tabular}{|c|c|c|}
\hline $\begin{array}{c}\lambda \\
(\mu \mathrm{m})\end{array}$ & $\begin{array}{l}\text { Flux } \\
\text { (mJy) }\end{array}$ & $\begin{array}{l}\text { Error } \\
(\mathrm{mJy})\end{array}$ \\
\hline 11.7.. & 175 & 10 \\
\hline 17.8 & 85 & 8 \\
\hline 450 & 130 & $40^{\mathrm{a}}$ \\
\hline 850 & 20 & $4^{\mathrm{a}}$ \\
\hline
\end{tabular}

${ }^{\text {a }}$ Calibration uncertainty.

to 0 ". 6 FWHM. Observations were conducted in the standard "chop/nod" mode, which involves switching between three sky positions using fast chopping of the secondary mirror and slower nodding of the telescope itself. This allows for effective subtraction of the very bright and variable thermal emission from the sky and telescope in the reduction process.

HD 107146 was well detected in both filters, with a formal signal-to-noise ratio of less than 15 for the $17.6 \mu \mathrm{m}$ data and less than 50 for the $11.7 \mu \mathrm{m}$ data. However, the seeing conditions limited the photometric precision, because of varying image quality from the science target to the standard stars. These conditions also prevented any useful constraints on any extended mid-IR emission from HD 107146. Photometric errors were first determined from the scatter in measurements done with apertures scaled by the FWHM of the images. We then added in quadrature an error term to account for seeing mismatch $(10 \mathrm{mJy}$ at $11.7 \mu \mathrm{m} ; 8 \mathrm{mJy}$ at $17.6 \mu \mathrm{m})$, estimated from analyzing curve-of-growth photometry for standard stars observed during the course of the entire night. The absolute flux calibration is based on the flux of Vega ( $\alpha$ Lyr) compiled by Tokunaga (1988). The LWS and SCUBA fluxes are listed in Table 1.

\section{RESULTS}

The resulting maps of the emission at 450 and $850 \mu \mathrm{m}$ are shown in Figure 1. There is a 2".9 offset between the two maps, as measured by Gaussian fits to the data clipped at the FWHM level. The centroid of the emission is consistent with the stellar position to within 4".4 $(450 \mu \mathrm{m})$ and 1".6 $(850 \mu \mathrm{m})$. It is not clear how significant such small offsets are, given the relatively low signal-to-noise ratio in the data and the possibility of systematic pointing errors as large as 2 ". 5 . No other source within this angular range of the star was apparent in the Digital Sky Survey, Two Micron All Sky Survey (2MASS), the Keck imaging at 11.7 and $17.8 \mu \mathrm{m}$, or the Faint Images of the Radio Sky at Twenty $\mathrm{cm}$ survey. In addition, Blain et al. (1999) predict 100-300 sources $\mathrm{deg}^{-2}$ with fluxes greater than $20 \mathrm{mJy}$ at $850 \mu \mathrm{m}$, implying a probability less than $1.4 \times 10^{-3}$ that an unrelated background object this bright would be found within 4 ."4 of the star. Since the probability of unrelated submillimeter emission is very low, we assume that the SCUBA source is a disk associated with the star and discuss its properties in this context.

Allowing for pointing errors, the resolutions of the maps are 14.5 at $850 \mu \mathrm{m}$ and $8^{\prime \prime}$ at $450 \mu \mathrm{m}$. The Gaussian fits give sizes of $15^{\prime \prime} 1 \times 14^{\prime \prime} .9$ and $133^{\prime \prime} 2 \times 10^{\prime \prime} .9$ (position angle $-35^{\circ}$ ), respectively. The elongation at $450 \mu \mathrm{m}$ is apparent at the halfpower level and is therefore not due to the JCMT beam pattern, which shows significant noncircularity only at the $10 \%$ level. The slight extension in the $450 \mu \mathrm{m}$ map is also 

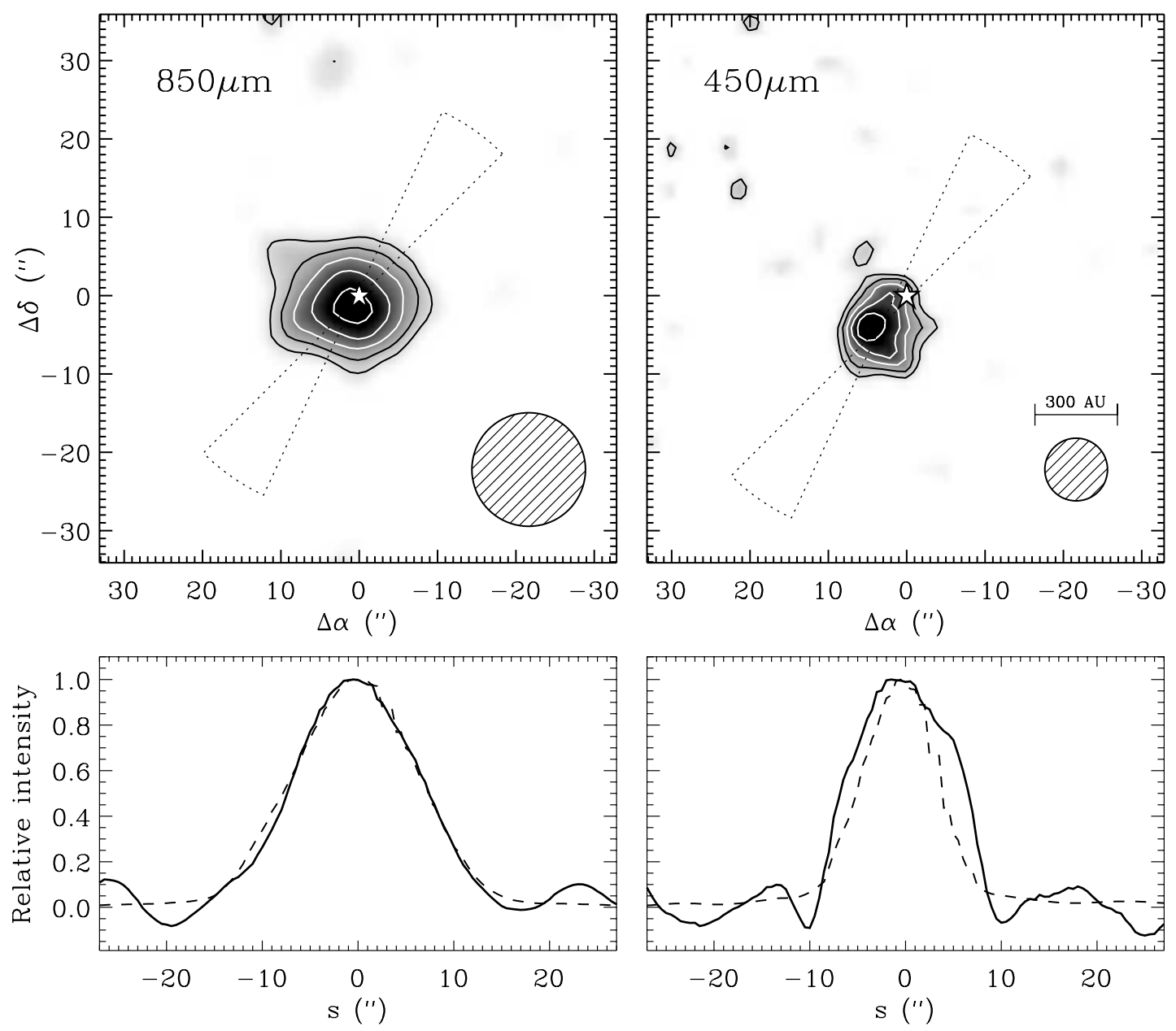

FIG. 1.-Top: SCUBA images of the dust emission around HD 107146. Coordinates are offset from the J2000.0 position of the star: epoch 2003.13, $\alpha=12^{\mathrm{h}} 19^{\mathrm{m}} 6 \mathrm{~s} 46, \delta=16^{\circ} 32^{\prime} 53^{\prime \prime} 4$, indicated by the star symbol. Top left: Emission at $850 \mu \mathrm{m}$. Contour levels begin at $2 \sigma$ and increment by $\sigma$, where $\sigma=4 \mathrm{mJy}$ beam $^{-1}$ is the noise in the map. The gray scale runs from 1 to $7 \sigma$. Top right: Emission at $450 \mu \mathrm{m}$; contours begin at $3 \sigma$ and increment by $\sigma$, where $\sigma=13 \mathrm{mJy}$ beam ${ }^{-1}$. The gray scale runs from 2 to $8 \sigma$. The beam sizes, 14.5 at $850 \mu \mathrm{m}$ and $8^{\prime \prime}$ at $450 \mu \mathrm{m}$, are indicated by the hashed circles in the lower right corner of each figure. Inspection shows that the half-power point of the $450 \mu \mathrm{m}$ image $(\sim 3.5 \sigma)$ is slightly greater than the beam size. Averaged cuts of the source and Mars are shown in the bottom panels for each wavelength. The cuts are centered on the peak of emission and averaged over position angles $-35^{\circ} \pm 10^{\circ}$, outlined by dotted lines in each image. The Mars profile is shown as the dashed line and is significantly narrower than that of HD 107146 in the higher resolution $450 \mu \mathrm{m}$ image. [See the electronic edition of the Journal for a color version of this figure.]

seen in crosscuts through the image along the major axis. To increase the signal-to-noise ratio, an average crosscut over a range of position angles $-35^{\circ} \pm 10^{\circ}$ centered on the peak of emission is shown for each wavelength in the bottom panels of Figure 1. The same crosscut averaging, shown as dashed lines, was performed for a map of Mars, taken from the last night of observations, February 19, when the Martian diameter was 5"7. We conclude that the disk around HD 107146 is marginally resolved at the $8^{\prime \prime}$ resolution of the $450 \mu \mathrm{m}$ data. Subtracting the beam size in quadrature from the Gaussian fit gives an angular size of $10^{\prime \prime} .5 \times 7$."4 for the disk, corresponding to $300 \mathrm{AU} \times 210 \mathrm{AU}$.

The fluxes measured from the LWS and SCUBA observations are listed in Table 1. The SED of the source, from optical to submillimeter wavelengths, is plotted in Figure 2. The $U B V R I$ photometry is from Landolt (1983), the near-IR fluxes in the $J H K$ bands are from $2 \mathrm{MASS}$, and the $10 \mu \mathrm{m}$ point is from Palomar observations (Metchev, Hillenbrand, \& Meyer 2004). IRAS fluxes were determined by color correcting the quoted values in the Faint Source catalog. The IRAS $12 \mu \mathrm{m}$ flux is consistent with the Keck $11.7 \mu \mathrm{m}$ observation and is not plotted. The stellar photosphere was fitted by a Kurucz model $\left(T_{\text {eff }}=5750 \mathrm{~K}, \log g=4.5\right.$, and solar metallicity) with a power-law extrapolation beyond $10 \mu \mathrm{m}$. Strong excess emission is apparent beyond $25 \mu \mathrm{m}$. In order to compare the properties of the excess with that detected from other debris disk systems, we fitted the disk SED using a single-temperature modified blackbody with emission efficiency $Q_{\lambda}=1-$ $\exp \left[-\left(\lambda_{0} / \lambda\right)^{\beta}\right]$, which has asymptotic behavior: $Q_{\lambda}=1$ for $\lambda \ll \lambda_{0}$ and $Q_{\lambda}=\left(\lambda / \lambda_{0}\right)^{\beta}$ for $\lambda \gg \lambda_{0}$. The critical wavelength, $\lambda_{0}$, was set to $100 \mu \mathrm{m}$ for consistency with the assumptions made in previous analyses of debris disk SEDs (Dent et al. 2000; Wyatt et al. 2003).

The parameters of the modified blackbody fit were measured using a least-squares fit to the data. Errors were estimated via fits to multiple simulations of the dust excess SED. Simulated data points were drawn from a Gaussian distribution, with the mean and standard deviation as determined by the observed data. The resulting distributions of best-fit parameter values are $T=51 \pm 4 \mathrm{~K}$ and $\beta=0.69 \pm 0.15$. Similarly low values of $\beta$ are found for other disks around main-sequence stars (Dent et al. 2000). The dust mass, based on the fitted flux at 


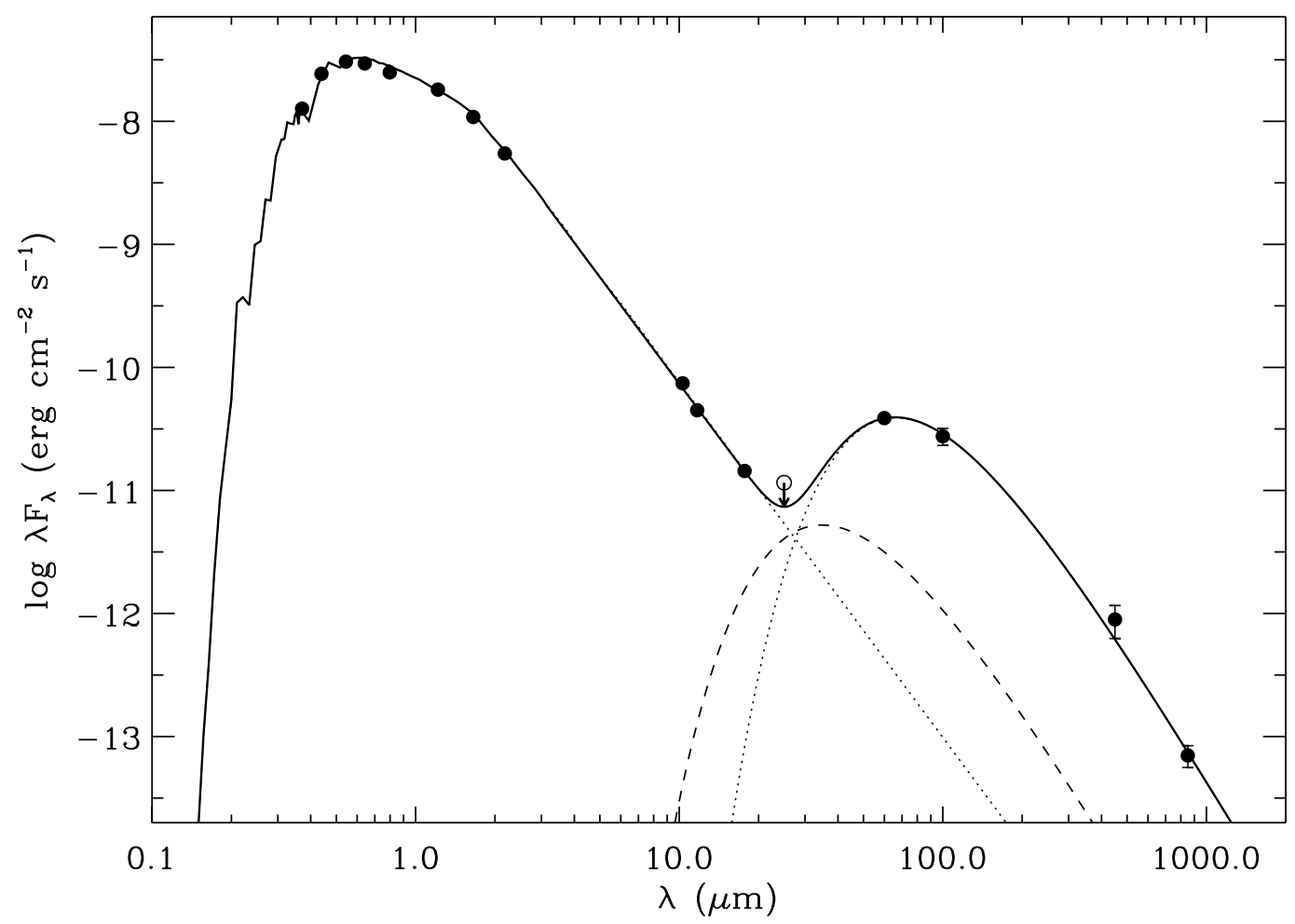

FIG. 2.-Optical to submillimeter SED of HD 107146. The open circle at $25 \mu \mathrm{m}$ represents an upper limit from the IRAS Faint Source catalog. All other points represent detections, with error bars shown when the error exceeds the symbol size. The double-peaked distribution is modeled as the sum of a Kurucz model of the stellar spectrum and a modified blackbody fit to the points longward of $25 \mu \mathrm{m}\left(T=51 \mathrm{~K}, M_{d}=0.10 M_{\oplus}, \beta=0.7\right)$. The individual contribution of each component is shown by the dotted lines and the sum of the two by the solid line. The dashed line shows the maximum allowable $T=100 \mathrm{~K}$ dust component that fits the constraint of the IRAS $25 \mu \mathrm{m}$ upper limit. This component is not included in the overall fit shown by the solid line.

$850 \mu \mathrm{m}$ and assuming a dust mass absorption coefficient of $\kappa_{850}=1.7 \mathrm{~cm}^{2} \mathrm{~g}^{-1}$, is $M_{d}=0.10 \pm 0.02 M_{\oplus}$. This value of $\kappa_{850}$ is chosen for consistency with Holland et al. (1998) and Greaves et al. (1998), but is on the high end of calculated values (Pollack et al. 1994). For the full range of $\kappa_{850}=$ 0.4-1.7 $\mathrm{cm}^{2} \mathrm{~g}^{-1}$ discussed in Pollack et al., the corresponding mass range is $M_{d}=0.10-0.43 M_{\oplus}$. As with all submillimeter observations, the inferred dust masses do not include a potentially dominant mass component that resides in larger bodies (grains and planetesimals) subtending a negligible solid angle. The mean parameter fit is shown in Figure 2.

The single-temperature fit is a simplification that is warranted by the small number of data points. Nevertheless, the large dip in the SED at $25 \mu \mathrm{m}$ imposes a strong limit on the mass of warmer dust that may be present in the system. To illustrate this constraint, a $T=100 \mathrm{~K}, \beta=0.7$ modified blackbody component was added to the SED fit and increased until the IRAS $25 \mu \mathrm{m}$ upper limit was exceeded. This maximum allowable contribution, which corresponds to a mass limit of $M_{d}(T=100 \mathrm{~K})=7 \times 10^{-4} M_{\oplus}$, is shown in Figure 2 but was not included in the overall fit shown. The Keck $18 \mu \mathrm{m}$ measurement tightly constrains the presence of still warmer dust to a $3 \sigma$ limit of $M_{d}(T=200 \mathrm{~K})<3 \times$ $10^{-5} M_{\oplus}$.

The limits on dust cooler than $50 \mathrm{~K}$ are less stringent, since such dust emits less per unit mass and does so at longer wavelengths. The minimum grain temperature is $23 \mathrm{~K}$ for blackbody particles at the measured outer radius of the disk, $150 \mathrm{AU}$ from the star. In practice, the effect of a range of cool dust temperatures is indistinguishable from changes in the wavelength dependence of the grain emissivity, parameterized by $\beta$. However, without changing the parameters of the fit in Figure 2, $0.13 M_{\oplus}$ of $23 \mathrm{~K}$ dust can be added before the $850 \mu \mathrm{m} 3 \sigma$ upper limit is exceeded. Thus, a significant fraction of the total dust mass may reside in cooler dust.

\section{DISCUSSION AND SUMMARY}

We have detected strong submillimeter excess emission from HD 107146, a G2 V star with an age estimated to lie in the range 80-200 Myr. Based on a fit to the SED of the system, the mass of the emitting dust is estimated to be $0.10 M_{\oplus}$ or larger. We also find that the disk is marginally resolved at $450 \mu \mathrm{m}$. The constraints placed by these observations on the mass, temperature, and physical extent of the dust in the system are compared in Table 2 with the properties of other well-studied debris disk systems.

Four of the six stars in Table 2 are A stars, a probable bias due to the relatively high luminosity of these systems at far-IR and submillimeter wavelengths. Nevertheless, despite the range in central star masses and luminosities, the disks have similar properties and follow several trends. The HD 107146 disk is quite massive, comparable in mass to the $\beta$ Pic disk. Although it lies noticeably above the trend of decreasing mass with age that is defined by HR 4796A, $\beta$ Pic, Fomalhaut, and Vega (Holland et al. 1998), it is within the scatter in the massage relation found for larger samples of dust disks (Wyatt et al. 2003). The fractional dust luminosity $\left(L_{d} / L_{*}\right)$ of HD 107146 is also large; it is half that of $\beta$ Pic, more than 10 times that of Fomalhaut, and consistent with the trend of decreasing $L_{d} / L_{*}$ with age (Spangler et al. 2001). Table 2 also indicates an apparent trend of decreasing outer disk radius with age. Perhaps this trend, admittedly of low statistical significance at 
TABLE 2

Properties of Spatially Resolved Submillimeter Disks around Class V Stars

\begin{tabular}{|c|c|c|c|c|c|c|c|c|c|c|}
\hline Source & Spectral Type & $\begin{array}{c}\text { Age } \\
(\mathrm{Myr})\end{array}$ & $\begin{array}{c}d \\
(\mathrm{pc})\end{array}$ & $\begin{array}{c}L_{*} \\
\left(L_{\odot}\right)\end{array}$ & $\begin{array}{l}L_{d} / L_{*} \\
\left(10^{-5}\right)\end{array}$ & $\begin{array}{c}R_{\text {in }} \\
(\mathrm{AU})\end{array}$ & $\begin{array}{l}R_{\text {out }} \\
(\mathrm{AU})\end{array}$ & $\begin{array}{c}T_{d} \\
(\mathrm{~K})\end{array}$ & $\begin{array}{c}M_{d} \\
\left(M_{\oplus}\right)\end{array}$ & References \\
\hline HR 4796A...... & A0 & $3-10$ & 67.1 & 21 & 500 & $\sim 50$ & $\sim 200$ & 110 & 0.250 & $1,2,3,4$ \\
\hline$\beta$ Pic............... & A5 & $10-100$ & 19.3 & 8.9 & 200 & $\sim 20$ & 210 & 85 & 0.096 & $5,6,7,8$ \\
\hline HD $107146 \ldots$. & G2 & $80-200$ & 28.5 & 1.1 & 120 & $>31$ & 150 & 51 & 0.100 & 9 \\
\hline Fomalhaut....... & A3 & $100-300$ & 7.7 & 13 & 10 & 60 & 160 & 40 & 0.018 & $5,6,7$ \\
\hline Vega...................... & A0 & $150-550$ & 7.8 & 60 & 2 & 70 & 90 & 80 & 0.009 & $5,6,7,10$ \\
\hline$\epsilon$ Eri................. & $\mathrm{K} 2$ & $\leq 10^{3}$ & 3.2 & 0.3 & 8 & 30 & 60 & 35 & 0.005 & $6,7,11$ \\
\hline
\end{tabular}

References.- (1) Jura et al. 1998; (2) Jayawardhana et al. 1998; (3) Greaves, Mannings, \& Holland 2000; (4) Schneider et al. 1999; (5) Holland et al. 1998; (6) Spangler et al. 2001; (7) Dent et al. 2000; (8) Lagage \& Pantin 1994; Pantin, Lagage, \& Artymowicz 1997; (9) this work; (10) Wilner et al. 2002; (11) Greaves et al. 1998.

present, will be verified when a larger number of debris disk systems have been spatially resolved.

We also find that the local minimum in the SED at $25 \mu \mathrm{m}$ places a strong limit on the amount of warm dust in the HD $107146 \operatorname{disk}, M_{d}(T=51 \mathrm{~K}) / M_{d}(T=100 \mathrm{~K}) \gtrsim 140$. This lack of warm dust implies that the disk does not extend all the way to the star. The dust temperature, and therefore the size of this inner hole, depends on the grain size distribution and optical properties. For grains that emit as blackbodies at $850 \mu \mathrm{m}, 51 \mathrm{~K}$ dust would lie at $31 \mathrm{AU}$ (Wyatt et al. 1999). This is a lower limit to the inner radius, since smaller grains could achieve this temperature at greater distances from the star. The other disks listed in Table 2 have inner holes of similar size, as determined from spatially resolved images in thermal emission or scattered light, and are comparable in size to the Kuiper Belt in our solar system.

Inner holes are not by themselves long lasting, because Poynting-Robertson drag will cause dust to spiral in from the outer disk onto the central star on $\sim 10$ Myr timescales. Such inward migration would be evident in the SED by the presence of warm dust (Jura et al. 1998). Thus, the existence of inner holes has been explained as a consequence of either the dynamical sweeping of an orbiting companion (e.g., Vega; Wilner et al. 2002) or the sublimation of icy grains (e.g., HR 4796A; Jura et al. 1998). Since water ice sublimates at temperatures greater than $100 \mathrm{~K}$ (Pollack et al. 1994), this explanation can not apply to the HD 107146 disk, where the shape of the SED places a strong limit on the mass of such warm dust. The alternative explanation, that the inner hole is dynamically maintained by a closely orbiting companion, would require a fairly low mass companion. The best current limit on the existence of close companions is from Palomar adaptive optics imaging by Metchev \& Hillenbrand (2004), who found no companions at detection limits of 11.2, 11.7, and 15.2 in absolute $K$-band magnitude at angular separations of $0.5,1^{\prime \prime}$, and $2^{\prime \prime}$, respectively. The corresponding mass limits for an age of $100 \mathrm{Myr}$ are approximately $30 M_{\mathrm{J}}, 25 M_{\mathrm{J}}$, and $10 M_{\mathrm{J}}$ (Burrows et al. 1997).

While it has been well recognized that the dynamical sculpting of disks by orbiting companions can produce inner holes and ringlike structures, recent studies have shown that the migration of dust in the presence of a residual gas disk can also induce ringlike structures in the dust distribution. For example, Takeuchi \& Artymowicz (2001) have shown that the dust structures seen in the HR 4796A and HD 141569A systems are qualitatively similar to those expected to result from the coupling between gas and dust in disks. However, orbiting companions may also induce significant departures from axisymmetry in the dust distribution (e.g., Liou \& Zook 1999; Ozernoy et al. 2000; Quillen \& Thorndike 2002; MoroMartín \& Malhotra 2002), whereas such nonaxisymmetric structures cannot be produced by dust migration. Thus, demonstrating the existence of asymmetries in the dust distribution, either in thermal emission or scattered light, as well as measuring the gas content of the HD 107146 disk, is needed to distinguish between these two possibilities.

At a distance of 28.5 pc, HD 107146 is relatively nearby. Compared to the other sources listed in Table 2, it is more distant than the debris disk systems that have been spatially resolved at submillimeter wavelengths ( $\beta$ Pic, Vega, Fomalhaut, and $\epsilon$ Eri), but it is at half the distance of HR 4796A. Since the HD 107146 disk is just resolved at the shortest operating wavelength of SCUBA, and the submillimeter excess is relatively bright, the Submillimeter Array should be able to map the morphology of the emitting dust in greater detail. Phase referencing will provide a more accurate absolute position, and such observations could determine whether the dust is distributed axisymmetrically (e.g., in a ring) or in a more asymmetric distribution. For example, these observations may confirm the marginal evidence for an offset between the stellar position and the peak of the $450 \mu \mathrm{m}$ emission found in the observations presented here (Fig. 1). The offset, if real, may result from an asymmetric dust distribution, as has been found for several of the other debris disk systems in Table 2.

The dust disk asymmetry can also be addressed by imaging the disk in scattered light. The possibility of detecting scattered light from Vega-like stars is of great interest, given the few such systems that have been detected thus far, despite extensive deep surveys (e.g., Kalas \& Jewitt 1996). Using the quantities listed in Table 2, we can make a rough estimate of the relative strengths of the scattered light from each disk. If we ignore the disk inclination and assume a similar dust grain size distribution and albedo, the scattering area will be proportional to $M_{d}$, and the flux of scattered light will be roughly proportional to $M_{d} L_{*} / R_{\text {out }}^{2} d^{2}$, where $d$ is the distance to the star and $R_{\text {out }}$ is the physical extent of the disk. The factor of $R_{\text {out }}^{2}$ arises in this expression because grains of a given size located farther from the star intercept less starlight. Hence, the surface brightness of the disk will be proportional to $M_{d} L_{*} / R_{\text {out }}^{4}$. A potentially more relevant comparison is of the contrast between the expected surface brightness from the disk and the flux from the star, which is proportional to $M_{d} d^{2} / R_{\text {out }}^{4}$ (e.g., Jura et al. 1998). For the disk around HD 107146, the expected surface brightness of the scattered light is half that of the disk around $\beta$ Pic, and the expected contrast is 9 times larger, suggesting that the scattered light from HD 107146 
might not only be quite bright but might also stand out against the glare of the star. Given the low dust temperature deduced for the HD 107146 disk $(<100 \mathrm{~K})$, the grains are expected to be icy with a high albedo, which favors the detection of scattered light.

Nevertheless, there are significant uncertainties associated with this estimate. Detecting reflected light will be more challenging if the disk is face-on rather than edge-on. In addition, the submillimeter measurements from which $M_{d}$ is derived are primarily sensitive to large grains $(\sim 100 \mu \mathrm{m})$, whereas the scattered-light observations will be particularly sensitive to much smaller grain sizes. Thus, scattered light from the HD 107146 disk will be weaker if the grain size distribution is significantly skewed to large grain sizes. Conversely, scatteredlight measurements can help constrain the grain size distribution of the disk (e.g., Artymowicz, Burrows, \& Paresce 1989).

Future spectroscopic observations of this disk with the Spitzer Space Telescope, particularly in the 30-40 $\mu \mathrm{m}$ range, will place additional constraints on the grain composition and size distribution (e.g., Wolf \& Hillenbrand 2003). The Spitzer Space Telescope is also expected to discover many more disks over a large range of ages and stellar masses, with consequent improvements for our understanding of the formation and evolution of planetary systems.

We thank Anneila Sargent for counseling during an extended review process, Hervé Aussel, Remo Tilanus, Iain Coulson, and Randy Campbell for advice on instrumentation, Eric Mamajek for helpful discussions on the age of HD 107146, and Alan Tokunaga for making the Keck time available. We acknowledge support from NSF grant AST 0324328 (J. P. W.), the Beatrice Watson Parrent Fellowship at the University of Hawaii and NASA grant HST-HF-01152.01 (M. C. L.), and NASA contract 1224768, administered through JPL (M. R. M., J. M. C., L. A. H.). This research has made use of the SIMBAD database.
Artymowicz, P., Burrows, C., \& Paresce, F. 1989, ApJ, 337, 494

Augereau, J. C., Lagrange, A. M., Moillet, D., \& Menard, F. 1999, A\&A, 350, L51

Aumann, H. H., et al. 1984, ApJ, 278, L23

Blain, A. W., Kneib, J.-P., Ivison, R. J., \& Smail, I. 1999, ApJ, 512, L87

Burrows, A., et al. 1997, ApJ, 491, 856

Dent, W. R. F., Walker, H. J., Holland, W. S., \& Greaves, J. S. 2000, MNRAS, 314, 702

Fisher, R. S., Telesco, C. M., Piña, R. K., Knacke, R. F., \& Wyatt, M. C. 2000, ApJ, 532, L141

Greaves, J. S., Mannings, V., \& Holland, W. S. 2000, Icarus, 143, 155

Greaves, J. S., et al. 1998, ApJ, 506, L133

Hatzes, A. P., et al. 2000, ApJ, 544, L145

Holland, W. S., et al. 1998, Nature, 392, 788 2003, ApJ, 582, 1141

Hollenbach, D. J., Yorke, H. W., \& Johnstone, D. 2000, in Protostars and Planets IV, ed. V. Mannings, A. P. Boss, \& S. S. Russell (Tucson: Univ. Arizona Press), 401

Jayawardhana, R., Fisher, S., Hartmann, L., Telesco, C., Piña, R., \& Fazio, G. 1998, ApJ, 503, L79

Jones, B. F., Fischer, D., Shetrone, M., \& Soderblom, D. R. 1997, AJ, 114, 352

Jura, M., Malkan, M., White, R., Telesco, C., Piña, R., \& Fisher, R. S. 1998, ApJ, 505, 897

Jura, M., Zuckerman, B., Becklin, E. E., \& Smith, R. C. 1993, ApJ, 418, L37

Kalas, P., \& Jewitt, D. 1996, AJ, 111, 1347

Koerner, D. W., Ressler, M. E., Werner, M. W., \& Backman, D. E. 1998, ApJ, 503, L83

Lagage, P. O., \& Pantin, E. 1994, Nature, 369, 628

Lagrange, A.-M., Backman, D. E., \& Artymowicz, P. 2000, in Protostars and Planets IV, ed. V. Mannings, A. P. Boss, \& S. S. Russell (Tucson: Univ. Arizona Press), 639

Landolt, A. U. 1983, AJ, 88, 853

Liou, J.-C., \& Zook, H. A. 1999, AJ, 118, 580

Marcy, G. W., Cochran, W. D., \& Mayor, M. 2000, in Protostars and Planets IV, ed. V. Mannings, A. P. Boss, \& S. S. Russell (Tucson: Univ. Arizona Press), 1285

Metchev, S. A., \& Hillenbrand, L. A. 2004, in ASP Conf. Ser. Debris Disks and the Formation of Planets, ed. L. Caroff \& D. Backman (San Francisco: ASP), in press

\section{EFERENCES}

Metchev, S. A., Hillenbrand, L. A., \& Meyer, M. R. 2004, ApJ, 600, 436

Micela, G., Sciortino, S., Harnden, F. R., Kashyap, V., Rosner, R., Prosser, C. F., Damiani, F., Stauffer, J., \& Caillault, J.-P. 1999, A\&A, 341, 751

Moro-Martin, A., \& Malhotra, R. 2002, AJ, 124, 2305

Ozernoy, L. M., Gorkavyi, N. N., Mather, J. C., \& Taidakova, T. T. 2000, ApJ, 537, L147

Pantin, E., Lagage, P. O., \& Artymowicz, P. 1997, A\&A, 327, 1123

Perryman, M. A. C., et al. 1997, A\&A, 323, L49

Pollack, J. B., Hollenbach, D., Beckwith, S., Simonelli, D. P., Roush, T., \& Fong, W. 1994, ApJ, 421, 615

Prosser, C. F., Randich, S., Stauffer, J. R., Schmitt, J. H. M. M., \& Simon, T. 1996, AJ, 112, 1570

Quillen, A. C., \& Thorndike, S. 2002, ApJ, 578, L149

Schneider, G., et al. 1999, ApJ, 513, L127

Silverstone, M. 2000, Ph.D. thesis, UCLA

Skrutskie, M. F., Dutkevich, D., Strom, S. E., Edwards, S., Strom, K. M., \& Shure, M. A. 1990, AJ, 99, 1187

Soderblom, D. R., Jones, B. F., Balachandran, S., Stauffer, J. R., Duncan, D. K., Fedele, S. B., \& Hudon, J. D. 1993, AJ, 106, 1059

Spangler, C., Sargent, A. I., Silverstone, M. D., Becklin, E. E., \& Zuckerman, B. 2001, ApJ, 555, 932

Sylvester, R. J., Dunkin, S. K., \& Barlow, M. J. 2001, MNRAS, 327, 133

Takeuchi, T., \& Artymowicz, P. 2001, ApJ, 557, 990

Telesco, C. M., et al. 2000, ApJ, 530, 329

Tokunaga, A. T. 1988, IRTF Photometry Manual (Honolulu: Univ. Hawaii Inst. Astron.)

Voges, W., et al. 1999, A\&A, 349, 389

Weinberger, A. J., et al. 1999, ApJ, 525, L53

Wichmann, R., Schmitt, J. H. M. M., \& Hubrig, S., 2003, A\&A, 399, 983

Wilner, D. J., Holman, M. J., Kuchner, M. J., \& Ho, P. T. P. 2002, ApJ, 569, L115

Wolf, S., \& Hillenbrand, L. A. 2003, ApJ, 596, 603

Wyatt, M. C., Dent, W. R. F., \& Greaves, J. S. 2003, MNRAS, 342, 876

Wyatt, M. C., Dermott, S. F., Telesco, C. M., Fisher, R. S., Grogan, K., Holmes, E. K., \& Piña, R. K. 1999, ApJ, 527, 918

Zuckerman, B., \& Becklin, E. E. 1993, ApJ, 414, 793 